cemoti $\begin{aligned} & \text { Cahiers d'études sur la Méditerranée } \\ & \text { orientale et le monde turco-iranien }\end{aligned}$

22 | 1996

Arabes et Iraniens

\title{
La redéfinition de la nation, de l'Etat et du citoyen
}

\section{Etienne COPEAUX}

\section{(2) OpenEdition}

Journals

Édition électronique

URL : https://journals.openedition.org/cemoti/150

DOI : $10.4000 /$ cemoti. 150

ISSN : $1777-5396$

Éditeur

AFEMOTI

Édition imprimée

Date de publication : 1 juin 1996

ISSN : 0764-9878

\section{Référence électronique}

Etienne COPEAUX, «La redéfinition de la nation, de l'Etat et du citoyen », Cahiers d'études sur la Méditerranée orientale et le monde turco-iranien [En ligne], 22 | 1996, mis en ligne le 04 mars 2005, consulté le 20 juin 2022. URL : http://journals.openedition.org/cemoti/150 ; DOI : https://doi.org/ 10.4000/cemoti. 150

Ce document a été généré automatiquement le 20 juin 2022.

Tous droits réservés 


\title{
La redéfinition de la nation, de l'Etat et du citoyen
}

\author{
Etienne COPEAUX
}

1 Un colloque organisé par le Centre de recherches sur les relations internationales de l'Université de Marmara et la Fondation Friedrich Ebert s'est tenu les 28 et 29 mars 1996 à Istanbul. Le propos portait sur "La redéfinition de la nation, de l'Etat et du citoyen". Une vingtaine de participants, venant d'horizons divers, se sont succédés à la tribune. Par son ambition même, le sujet retenu portait en lui le risque de verser dans des propos très généraux; mais, grâce à une série de communications précises, ce risque a été en grande partie évité.

2 La première demi-journée du colloque a été consacrée à l'arrière-plan historique de la genèse de l'Etat-nation et de la citoyenneté. Un long développement de Kemâli Saybasili sur la politique et l'histoire a introduit une étude du cas de la Turquie, où Füsun Üstel a montré, de manière précise et argumentée, comment l'enseignement de l'instruction civique, à partir de 1924, a formé le "profil officiel du citoyen", évoluant au fil des décennies vers un modèle de "citoyenneté naturelle" indexée sur l'ethnicité turque et l'islam et provoquant de ce fait un malaise grandissant sur le plan identitaire ${ }^{1}$.

3 La première session de l'après-midi a promené l'assistance des Caraïbes au MoyenOrient en passant par l'Afrique; il s'agissait d'examiner le processus de formation d'Etats-nations post-coloniaux. Ralph Premdas (Toronto) a évoqué les réponses de l'Etat face au sécessionisme, dans les cas de Sri-Lanka et de la Papouasie et s'est interrogé sur la validité de la solution autonomiste ou régionaliste. J.-L. Balans (Université de Marmara) a évoqué l'existence d'un début de crise identitaire africaine en insistant sur le caractère artificiel et relativement récent de certaines "ethnies". Les contributions de David McDowall et Barry Rubin (Bar-Ilan, Israël) sur le Moyen-Orient et Israël ont provoqué un débat très animé; le point de vue de ce dernier, qui a bâti son exposé comme si les Arabes d'Israël étaient une "minorité" comme une autre et comme si les Juifs formaient une communauté ethnique soudée et homogène, a entraîné des 
réactions si vigoureuses que la tranquille autorité du président de séance, Mete Tunçay, s'est révélée bien utile.

4 Après la pose, on a débattu de l'espace post-communiste; selon Chris Rumford (IKV, Istanbul), la chute des régimes communistes en Europe a entraîné l'affaiblissement du lien entre l'Etat et la nation, et la principale menace viendrait maintenant du régionalisme, "largement déterminé par l'ethnicité" et élément primordial de l'identité collective actuelle. Sule Kut (Marmara) a présenté les cas de la Bosnie-Herzégovine et de la Macédoine, dont les situations politico-ethniques étaient semblables et dont les évolutions ont été radicalement inverses, pour l'instant. Enfin, Nadir Devlet (Marmara), spécialiste des Tatars, a brossé un tableau de la situation dans les régions turcophones de l'espace post-soviétique, s'interrogeant sur l'évolution de l'identité "turcique" alors que l'identité "nationale" des républiques turcophones se renforce.

5 La seconde journée du colloque portait sur "Citoyenneté et identité dans un cadre mondialisé" (global world, küresellesen dünya). A. Samarasinghe (Tulane Univ., USA) a traité de l'aspect économique des conflits ethniques, en s'appuyant sur le cas SriLankais, avec une double approche : quel est le rôle des inégalités économiques entre les groupes ethniques? Et quelles sont les conséquences politiques du coût économique des conflits interethniques? L'une des communications les plus attendues était sans conteste celle de Martin van Bruinessen. Evoquant tout d'abord sur les différences qui se sont creusées entre les Kurdes de Turquie, d'Irak et d'Iran -grâce à l'efficacité de l'école, de l'armée et de la presse dans la formation des mentalités- il a insisté sur le fait que les Kurdes de Turquie sont des Turcs qui pensent en Turcs. En conséquence, il perçoit le PKK comme un mouvement néo-kémaliste qui aborde le problème kurde avec une méthodologie semblable à celle qui prévalait en Turquie dans les années trente. Résumant les principaux changements qui ont eu lieu, à ses yeux, depuis 1990, Martin van Bruinessen a évoqué la montée du PKK, qu'il définit comme un produit indirect de la politique turque, puisque celle-ci n'a pas donné d'autre alternative à la population; les migrations vers les pays voisins et vers l'Europe, qui ont hâté la formation d'éléments intégrateurs dans la population kurde; la montée d'une prise de conscience kurde dans les populations vivant en Europe, conscience qui n'existait que très peu vers 1980; l'émergence, en Europe, d'une langue kurde "standard", notamment dans son expression littéraire; enfin, la formation en Turquie d'une sous-classe due aux migrations, non intégrée dans la société de l'ouest et dont la croissance pourrait être à l'origine d'événements dramatiques du type de ceux de Gaziosmanpasa en mars 1995. Dans l'exposé suivant, Günay Göksu Özdogan (Marmara) a offert une réflexion générale sur les interactions et tensions entre les identités culturelles et politiques, le problème de la place de l'ethnicité dans la définition de la citoyenneté.

6 La session suivante a été consacrée à des questions qui surgissent de certains cas de reconstruction de l'Etat-nation. Deniz Vardar (Marmara) a traité de l'impact de la candidature turque à l'Union Européenne sur l'identité et la citoyenneté, en tenant compte de l'influence des politiques migratoires européennes et des débats sur la citoyenneté en Turquie. Birgit Brandt (Université de Warwick) s'est également penchée sur les rapports turco-européens à travers le cas de la migration en Allemagne et les problèmes spécifiques qu'on y rencontre, puisque la citoyenneté est définie par l'ethnicité (cas unique en Europe); la multi-ethnicité de la population n'y est pratiquement pas prise en compte officiellement, et l'immigration n'y est pas l'objet d'une politique à long terme. L'exposé de Daniele Conversi (Syracuse University, USA) 
portait sur le cas de l'Espagne qui est passée d'une dictature centralisée à une démocratie fédérale; le problème basque, avec ses expressions violentes, et la résolution pacifique du problème catalan sont souvent évoqués dans le débat politique turc à propos du problème kurde, et l'analyse du cas espagnol présentait un grand intérêt pour l'appréhension des problèmes de la transition démocratique lorsqu'elle se complique d'un problème ethnique. Concluant la séance, le vigoureux exposé de Majid al-Haj (Haifa, Israël) sur l'éducation pluri-culturelle en Israël a souligné l'asymétrie qui y prévaut, puisque l'enseignement est largement ethnocentrique dans les écoles juives; l'intervenant s'est demandé comment on pouvait créer des "ponts culturels" entre les mondes israëlien et arabe, et comment concevoir un respect mutuel (shared civility) entre les citoyens juifs et arabes d'Israël ${ }^{2}$.

7 Le colloque s'est terminé par des réflexions sur la Turquie, en un panel réunissant des intellectuels turcs. Ahmet Insel (Paris-I) a posé le problème de la possibilité d'une diversification de l'identité centrale et unique, dans un cadre démocratique; parlant de l'islam turc, il a souligné sa forte dimension nationale (ulusal), son encadrement en des structures nationales, ce fait étant fortement illustré par les organisations musulmanes d'Europe. Ahmet Insel se montre très circonspect dans l'emploi des mots; dans ses chroniques de Yeni Yüzyl, il utilise volontiers, pour désigner les habitants de la Turquie, le terme de Türkiyeli, terme qui permet d'englober sans ambiguïté toutes les cultures présentes sur ce sol; ici, il a préféré parler d'appartenance (aidiyet) plutôt que d'identité et a souligné, parmi les obstacles à la diversification, la structure néo-féodale de la Turquie, ainsi que la psychose de l'encerclement, l'éternelle croyance -entretenue par l'Etat et les media- en un ennemi potentiel, qui a fini par produire un nationalisme très populaire s'exprimant dans la vie de tous les jours (gösteri milliyetçilik, sahne milliyetçilik). Ayse Kadogu (Université de Bilkent) a montré qu'en Turquie l'Etat a des particularités indépendantes de la nation puisqu'il existait avant la nation; elle a soulevé la question du rôle de l'éducation et des manuels scolaires, qui ont créé une "épistémologie républicaine". Ayhan Kaya (Université de Warwick) est revenu sur le nationalisme populaire, le "nationalisme théâtral" (sahne milliyetçilik) tel qu'il est mis en scène au sein de groupes de musique pop par les Turcs de la seconde génération en Allemagne. Ces sociétés synchrétiques se fondent autour d'un "créole" turco-germano-anglais, résultent d'un "collage culturel", de la recherche d'une culture populaire; mais il existe un mouvement d'aller-retour dialectique entre cette nouvelle culture et le retour à l'"authenticité" anatolienne; on assiste ici à l'émergence d'un nationalisme de minorité, en réaction à la xénophobie ambiante. Après une intervention de Fuat Keyman (Bilkent) sur le thème de l'universalisme et de la démocratie radicale, Baskin Oran (Université d'Ankara) est revenu sur la question kurde. En un exposé très didactique, il s'est interrogé sur l'impact des points objectifs de l'identité kurde (le nombre, la continuité historique) au regard des points subjectifs tels que la notion de majorité (notion ambiguë du fait des migrations internes) ou la motivation, elle-même fonction de la géopolitique, de la force de l'irrédentisme, de la conjoncture internationale. Rappelant que tous les nationalismes ont commencé par un nationalisme culturel, il a souligné la naissance, là aussi, d'un "nationalisme de minorité".

8 Si le thème des débats portait en principe sur un phénomène mondial, on ne pouvait s'abstraire du lieu et des circonstances: nous étions en Turquie, à un moment où aucune solution n'était en vue pour le problème kurde, et la majorité des intervenants étaient turcs. C'est dire que le problème kurde a plané sur l'assemblée durant ces deux jours, même si, bien souvent, cette préoccupation majeure de la société turque actuelle 
n'était pas explicitement évoquée. Il a été question de "régulation des conflits ethniques", de "crise de l'identité", de "l'avenir des minorités", du "régionalisme et de l'ethnicité", du "problème identitaire", de l'"impact économique des conflits ethniques", de l'"enseignement pluri-ethnique", et ces interventions portant sur des domaines géographiques très variés, il s'agissait de replacer le problème kurde dans une perspective élargie et une problématique comparative. Les deux exposés concernant explicitement la question kurde (Martin van Bruinessen et Baskin Oran) ont été suivis avec une attention particulière.

Il est nécessaire de faire ici une autre remarque d'ensemble : il existe une propension, chez certains intervenants, à se limiter à des propos extrêmement généraux, sous prétexte de théorisation. Parler de citoyenneté, de nation et d'Etat sans s'appuyer sur des exemples concrets est un exercice difficile. Si l'on se limite à se demander quelle étiquette convient à tel phénomène réel et complexe, qui requerrait avant tout des analyses serrées, on court le risque de jouer sur les mots. Inversement, les interventions précises, portant sur un sujet bien délimité, reposant sur une problématique et une approche clairement exposées, ont été bienvenues et ont toujours obtenu un intérêt soutenu du public.

\section{NOTES}

1. L'exposé de Füsun Üstel a été publié dans Yeni Safak, 14 avril 1996.

2. Majid Al-Haj vient de publier Education, Empowerment and Control. The Case of the Arabic Israël, New York, State University of New York Press, 1995, XIV, 249 p.

\section{RÉSUMÉS}

Si le thème des débats portait en principe sur un phénomène mondial, on ne pouvait s'abstraire du lieu et des circonstances : nous étions en Turquie, à un moment où aucune solution n'était en vue pour le problème kurde, et la majorité des intervenants étaient turcs. C'est dire que le problème kurde a plané sur l'assemblée durant ces deux jours, même si, bien souvent, cette préoccupation majeure de la société turque actuelle n'était pas explicitement évoquée. Il a été question de "régulation des conflits ethniques", de "crise de l'identité », de "l'avenir des minorités ", du "régionalisme et de l'ethnicité », du "problème identitaire », de l'« impact économique des conflits ethniques », de l' "enseignement pluri-ethnique », et ces interventions portant sur des domaines géographiques très variés, il s'agissait de replacer le problème kurde dans une perspective élargie et une problématique comparative. Les deux exposés concernant explicitement la question kurde (Martin van Bruinessen et Baskýn Oran) ont été suivis avec une attention particulière. 